\title{
Review Article \\ TAMH: A Useful In Vitro Model for Assessing Hepatotoxic Mechanisms
}

\author{
Madison Davis and Brendan D. Stamper \\ Pacific University School of Pharmacy, 222 SE 8th Avenue No. 451, Hillsboro, OR 97123, USA \\ Correspondence should be addressed to Madison Davis; madisondavis@pacificu.edu
}

Received 22 September 2016; Revised 10 November 2016; Accepted 24 November 2016

Academic Editor: Hemant Kumar

Copyright (C) 2016 M. Davis and B. D. Stamper. This is an open access article distributed under the Creative Commons Attribution License, which permits unrestricted use, distribution, and reproduction in any medium, provided the original work is properly cited.

In vitro models for hepatotoxicity can be useful tools to predict in vivo responses. In this review, we discuss the use of the transforming growth factor- $\alpha$ transgenic mouse hepatocyte (TAMH) cell line, which is an attractive model to study drug-induced liver injury due to its ability to retain a stable phenotype and express drug-metabolizing enzymes. Hepatotoxicity involves damage to the liver and is often associated with chemical exposure. Since the liver is a major site for drug metabolism, drug-induced liver injury is a serious health concern for certain agents. At the molecular level, various mechanisms may protect or harm the liver during drug-induced hepatocellular injury including signaling pathways and endogenous factors (e.g., Bcl-2, GSH, Nrf2, or MAPK). The interplay between these and other pathways in the hepatocyte can change upon drug or drug metabolite exposure leading to intracellular stress and eventually cell death and liver injury. This review focuses on mechanistic studies investigating drug-induced toxicity in the TAMH line and how alterations to hepatotoxic mechanisms in this model relate to the in vivo situation. The agents discussed herein include acetaminophen (APAP), tetrafluoroethylcysteine (TFEC), flutamide, PD0325901, lapatinib, and flupirtine.

\section{Introduction}

Over twenty years ago, a cell line was developed from longterm primary cultures of transgenic mouse hepatocytes that constitutively expressed transforming growth factor alpha (TGF- $\alpha$ ) [1]. This cell line is commonly referred to as TAMH (TGF- $\alpha$ transgenic mouse hepatocytes). It strongly expresses human TGF- $\alpha$ mRNA and is capable of proliferating without exogenous growth factors. In primary culture, these cells exhibit DNA synthesis and undergo a pattern of TGF- $\alpha$ induction similar to nontransgenic hepatocytes containing exogenous growth factors. High expression of TGF- $\alpha$ in murine hepatocytes activates an autocrine growth stimulatory loop allowing for autonomous replication [1]. On a molecular level, TGF- $\alpha$ is known to contribute to hepatocyte proliferation and transformation primarily during fetal, regenerative, and neoplastic growth [2]. Early studies of the TAMH model confirmed that high TGF- $\alpha$ expression promoted proliferation and neoplasia, but that overexpression was not the sole cause of carcinogenesis [1]. Furthermore,
DNA replication in TAMH can be inhibited using interferon gamma in combination with either tumor necrosis factor or lipopolysaccharide, similar to other cell lines of mouse origin [3]. The Fausto lab was able to determine that the TAMH cell line is a reliable model for hepatocarcinogenesis, demonstrated by their ability to transform in vitro and form differentiated tumors that exhibit characteristics similar to that of liver tumors in transgenic mice [1]. The utility of the TAMH cell line has expanded from this initial work into a valuable in vitro tool to assess mechanisms associated with hepatotoxicity. Currently, six agents have been studied at various depths in TAMH including acetaminophen (APAP), tetrafluoroethylcysteine (TFEC), flutamide, PD0325901, lapatinib, and flupirtine (Table 1). Prior to the evaluation of hepatotoxicity studies in the TAMH model, endogenous factors that contribute to cytoprotective and cytotoxic pathways during hepatocellular injury will be reviewed. These factors include glutathione (GSH), the B-cell lymphoma 2 (Bcl-2) protein family, mitogen-activated protein kinases (MAPKs), and nuclear factor- (erythroid-derived 2-) like 2 (Nrf2). 
TABLE 1: List of compounds investigated in TAMH and their general effects.

\begin{tabular}{|c|c|c|c|}
\hline Agent & Drug class & Pathways affected & Reference(s) \\
\hline APAP & Analgesic & $\begin{array}{l}\text { (i) ATP depletion } \\
\text { (ii) GSH depletion } \\
\text { (iii) Induces Jnk activation } \\
\text { (iv) Upregulation of ETC protein components } \\
\text { (v) Activation of p53 signaling }\end{array}$ & {$[31,39,40,44]$} \\
\hline TFEC & N/A; fluorocarbon metabolite & $\begin{array}{l}\text { (i) Nrf2 induction and increased expression of ARE response genes } \\
\text { (ii) Induction of ER-stress response genes } \\
\text { (iii) Cell death is Bax-mediated (antagonized by Bcl-xl } \\
\text { overexpression) } \\
\text { (iv) Upregulation of Hsps }\end{array}$ & {$[12,55,81]$} \\
\hline Flutamide & Antiandrogen & $\begin{array}{l}\text { (i) Upregulation of Nrf2 response genes } \\
\text { (ii) Downregulation of fatty acid } \beta \text {-oxidation genes Mitochondrial } \\
\text { dysfunction (ATP depletion, complex I inhibition) }\end{array}$ & [61] \\
\hline PD035901 & MEK inhibitor & $\begin{array}{l}\text { (i) Inhibits ERK activation } \\
\text { (ii) Inhibited cell growth }\end{array}$ & [63] \\
\hline Lapatinib & Tyrosine kinase inhibitor & (i) Cyp3a induction (dexamethasone) increased cell death & [67] \\
\hline Flupirtine & Analgesic & (i) Toxicity not directly linked to oxidative processes & {$[72]$} \\
\hline
\end{tabular}

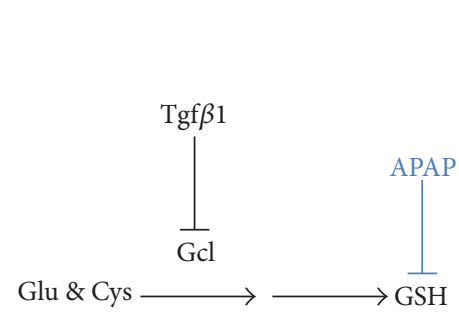

(a)

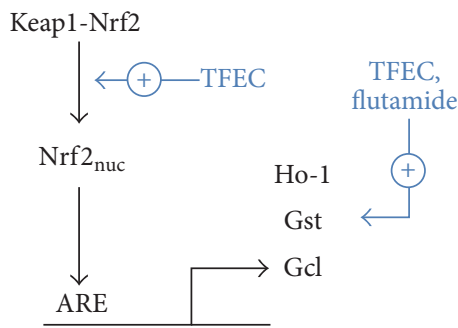

(c)

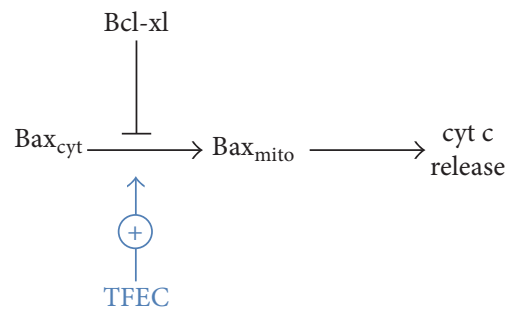

(b)

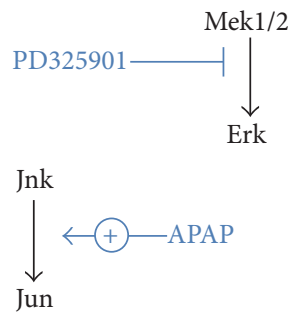

(d)

FIGURE 1: Simplified mechanistic schematics of signaling pathways affected by various agents in TAMH: (a) glutathione, (b) Bcl-2 protein family, (c) Nrf2 pathway, and (d) MAPK signaling. Please refer to the text for definitions for the included abbreviations.

\section{Pathways}

2.1. Glutathione. Intracellular GSH production and utilization are an essential cytoprotective pathway serving as an antioxidant defense system known to protect the liver from toxic insults (Figure 1(a)) [4]. The first and rate-limiting step in GSH biosynthesis is catalyzed by glutamate cysteine ligase $(\mathrm{Gcl})$, which contains a glutamate cysteine ligase catalytic subunit (Gclc) [5]. Downregulation of Gclc gene expression results in decreased Gcl activity and depletion of intracellular GSH. Mechanistic studies in the TAMH line identified transforming growth factor $\beta 1(\operatorname{Tgf} \beta 1)$ as a factor capable of modulating Gclc activity in this manner [6]. $\operatorname{Tgf} \beta 1$ has been shown to be a potent inhibitor of hepatocyte proliferation and induces apoptosis in TAMH [6, 7]. Cell death was found to correlate with intracellular GSH depletion through caspase-mediated cleavage of Gclc, loss of Gclc protein expression, and reduction in Gcl activity leading to a temporary disruption in intracellular GSH production [6]. GSH deficiency in TAMH has also been shown to sensitize cells to the effects of tumor necrosis factor, suggesting that TAMH is a good model for studying hepatocellular injury preceded by oxidative stress [8].

2.2. Bcl-2 Protein Family. The Bcl-2 family of proteins, including $\mathrm{Bcl}-2, \mathrm{Bcl}-2$ associated $\mathrm{X}$ protein (Bax), and B-cell lymphoma-extra large $(\mathrm{Bcl}-\mathrm{xL})$, play a central yet complex 
role in early determinations of cell fate (Figure 1(b)) [9]. For example, proapoptotic Bax is constitutively expressed in the cytosol but translocates to the mitochondria inducing pore formation during drug-induced toxic events [10]. Conversely, overexpression of antiapoptotic Bcl-xL has been shown to be capable of inhibiting mechanisms associated with drug-induced apoptosis [11]. In TAMH, sensitivity to $\operatorname{Tgf} \beta 1$ induced cell death is regulated by the level of $\mathrm{Bcl}-\mathrm{xL}$ protein that is expressed. Overexpression of Bcl-xL was shown to prevent $\operatorname{Tgf} \beta 1$-induced apoptosis by inhibiting caspase activity and cell death, leading to the observation that Bcl-xL overexpression correlates highly with cell viability [12]. The overexpression of $\mathrm{Bcl}-\mathrm{xL}$ in TAMH is believed to shift energy utilization from oxidative phosphorylation to glycolysis, and acute inhibition of cells overexpressing Bcl-xl led to progressive mitochondrial accumulation of reduced $\mathrm{NAD}(\mathrm{P}) \mathrm{H}$ and the generation of reactive oxygen species (ROS) [13]. Production of ROS has been shown to be prominent during drug-induced hepatotoxic events and a mediator of cell fate through disruption of mitochondrial function [14].

2.3. Nrf2 Pathway. Nrf2 is a stress responsive transcription factor that is particularly important during times of high oxidative stress through its ability to regulate the expression of antioxidant proteins and phase II enzymes, such as glutathione transferases, heme oxygenase-1 (Ho-1), glutathione S-transferase (Gst), and Gcl (Figure 1(c)) [15]. Nrf2 is constitutively expressed and localized to the cytosol in a repressed state with Kelch Like ECH Associated Protein 1 (Keap1). During instances of oxidative and nitrosative stress, Nrf2 dissociates with Keapl through modifications to Keapl's redox-sensitive cysteine-rich surface. Nrf2 then translocates to the nucleus where it associates with antioxidant response element consensus sequences on the promoter regions of its gene targets to combat oxidative stress through the expression of cytoprotective defense genes. Nrf2 has also been shown to crosstalk with MAPK signaling as MAPKs have been shown to indirectly mediate Nrf2 protein synthesis and play a role in direct phosphorylation of Nrf2 itself [16, 17].

2.4. MAPK Signaling. MAPK proteins are involved in a wide range of physiological processes such as proliferation, differentiation, and cell survival by triggering a phosphorelay cascade to the nucleus where gene expression is modulated [18]. They are organized into various groupings based on their sequence homology such as extracellular signal-related kinases (ERKs), c-Jun N-terminal kinases (JNKs), and p38s (Figure 1(d)) [19]. Studies have indicated that patterns of MAPK pathway upregulation occur specifically in hepatic carcinoma and not in normal hepatic tissue, thereby suggesting a unique mechanism for uncontrolled growth [20-23]. TGF- $\alpha$ is an MAPK pathway stimulator, initially signaling through the epidermal growth factor receptor, which then signals using the ERK pathway [24]. Treatment with MAPK modulators, such as JNK inhibitors, has been shown to protect hepatocytes against injury through various mechanisms including inhibition of Jun protooncogene (c-jun) phosphorylation and inhibition of the mitochondrial permeability transition $[25,26]$.

\section{Agents}

3.1. Acetaminophen. Acetaminophen ( $\mathrm{N}$-acetyl-para-aminophenol; APAP) is a widely used analgesic and antipyretic agent. However, both unintentional and intentional overdoses represent a clinically relevant contribution to cases of drug-induced liver injury [27, 28]. In overdose situations, higher concentrations of APAP are bioactivated in the endoplasmic reticulum by the cytochrome $\mathrm{P} 450$ oxidase enzyme system to $\mathrm{N}$-acetyl-paraquinoneimine (NAPQI), a reactive metabolite responsible for the covalent modification of target proteins, and cell death [29]. GSH has been shown to detoxify NAPQI through formation of an APAP-GSH conjugate. However, GSH is depleted in overdose situations due to excessive NAPQI formation, which allows NAPQI to bind to proteins and form adducts [30]. The TAMH cell line retains CYP450 protein expression capable of forming NAPQI (e.g., CYP2E1 and CYP3A) and possesses other markers that are characteristic of APAP-mediated cell death, which make it a useful cell culture model to study APAP-induced hepatocellular injury $[31,32]$.

In TAMH, multiorganellar collapse was evident following exposure to high APAP concentrations resulting in the inhibition of cellular proteasome activity and cell death that only partially resembled apoptosis with additional distinctive features consistent with long-term necrosis [31]. However, treatment with an APAP regioisomer, $3^{\prime}$ hydroxyacetanilide (N-acetyl-meta-aminophenol; AMAP), proved to be relatively nonhepatotoxic and had no effect on proteasome stability. The differential toxicities observed between these two isomers in the TAMH cell line were similar to early in vivo observations in mice [33]. Over time, TAMH has been a valuable tool in understanding mechanisms associated with APAP-induced toxicity through the utilization of the structure-toxicity relationship with AMAP. For example, AMAP-protein adduct formation was discovered later to reside mainly in the cytosol and endoplasmic reticulum, whereas APAP-protein adducts are commonly found in the mitochondria [34-37]. This led to a hypothesis that "lethal," less reactive, longer-lived APAP metabolites are able to migrate and bind to mitochondrial proteins whereas "nonlethal," more reactive, shorter-lived AMAP metabolites bind closer to their site of bioactivation. However, this hypothesis could be mouse model specific as a recent study in primary human hepatocytes found extensive mitochondrial protein adducts following exposure to AMAP [38].

Subsequent comparative studies using APAP and AMAP have identified numerous mechanisms associated specifically with APAP exposure. For example, protein-based studies have identified numerous changes associated with APAPinduced toxicity. These changes include potential compensatory mechanisms such as decreased P450 expression, upregulation of the mitochondrial isoform of superoxide dismutase, and upregulation of proteins associated with the electron transport chain and the mitochondrial permeability transition pore [39]. APAP has also been shown to deplete mitochondrial GSH in TAMH (Figure 1(a)) [40]. Disruption of these types of mitochondrial processes is well-established as a hallmark of APAP-induced liver injury, which validates these 
observations in the TAMH line [41, 42]. APAP-specific upregulation of Atf3 has been observed in TAMH [31, 40], a cellular event also observed in vivo [43]. Results from a comparative microarray study investigating APAP- and AMAP-induced effects across multiple model systems and platforms identified p53 signaling as significantly differentially regulated and unique to APAP exposure [44]. p53 is a tumor suppressor protein regulated by posttranslational modifications (e.g., phosphorylation, acetylation, and ubiquitination) and plays a critical role in determining cell fate during times of cell stress and DNA damage [45]. Immunoblotting experiments found total p53 levels to be marginally higher following APAP treatment compared to either the control or AMAP treatments. However, APAP exposure led to increased phosphorylation of p53 at both ser-15 and ser-392 residues suggesting that APAP not only induces a cell stress response but also enhances overall p53-mediated transcriptional activity [44]. APAPinduced phosphorylation of p53 has also been observed in murine liver [46] as well as other cell types such as glioma cells [47].

Finally, modulation of Jnk signaling following exposure to hepatotoxic APAP concentrations has been implicated as a key pathway during APAP-induced hepatocellular injury in TAMH [40]. Comparative transcriptomics identified increased expression of c-jun, a gene target of Jnk (Figure $1(\mathrm{~d})$ ). At the protein level, both c-jun and Jnk phosphorylation were observed at early time points as well. Similar transcript upregulation and posttranslational regulation were not observed following AMAP treatment; however, increased phosphorylation of Erk1/2 was seen early on. These observations have led to the hypothesis that alterations to MAPK signaling might be a key component behind the different toxicological outcomes for each regioisomer [40]. These observations are well-supported in the literature as Jnk signaling has long been known to contribute to the pathophysiology associated with APAP-induced hepatocellular injury in various human and rodent systems [48]. In fact, Jnk phosphorylation and mitochondrial translocation have been postulated as a "second hit," which follows GSH depletion and protein adduct formation serving to amplify the toxic response to APAP $[49,50]$.

3.2. TFEC. Tetrafluoroethylcysteine (TFEC) is a major metabolite of tetrafluoroethylene (widely used as a precursor for TEFLON coating) and a potential nephrotoxic agent $[51,52]$. TFEC bioactivation occurs in the mitochondria where cleavage at the beta position by pyridoxal-dependent aminotransferases occurs [53]. This reactive metabolite is thought to form covalent adducts to local proteins in the mitochondria, including heat shock proteins (HSPs) and those involved in the citric acid cycle [54]. These adducts are known to form in vivo and are thought to play a role in the kidney and liver damage that is observed [55]. In a microarray study investigating the effects of TFEC exposure, many genes associated with the homeostatic stress response, metabolism, apoptosis, transporters, and transcriptional regulation were upregulated, whereas cell cycling and immune response genes were downregulated [55]. Although the TAMH cell line is not of renal origin, TFEC does cause slight hepatotoxicity and the cell line has sufficient bioactivation capacity to reproduce key aspects of TFEC-induced toxicity $[31,51,54]$.

After exposure to toxicologically relevant TFEC concentrations, the TAMH model demonstrated inhibition of aconitase and $\alpha$-ketoglutarate dehydrogenase as well as the associated depletion of $\mathrm{NAD}(\mathrm{P}) \mathrm{H}$ and ATP biosynthesis, similar to what is observed in vivo $[54,56]$. Furthermore, Nrf2 was rapidly induced and induction was sustained for up to six hours resulting in increased expression of ARE responsive genes, especially heme oxygenase-1 and glutathione Stransferase (Figure 1(c)). Quantification of Nrf2 following TFEC exposure identified rapid mobilization from the cytosol to the nucleus. This was associated with an early endoplasmic reticular (ER) stress response and time dependent upregulation of ER-response genes, suggesting a potential alternative pathway for Nrf2 phosphorylation through ERmediated protein kinases [56]. Lastly, Bcl-xL overexpression, which led to the suppression of Bax movement to the mitochondria, protected against TFEC-induced injury (Figure 1(b)) [12]. TFEC exposure also led to a unique HSP regulation profile [55]. Interestingly, a similar pattern is not expressed when TAMH cells were treated with other hepatotoxicants. This unexpected cytosolic HSP response can be assumed to be an early signaling event from the mitochondria directed towards the cytosol where the activation of specific transcription factors can be initiated. In addition, microarray analysis in TAMH cells treated with TFEC revealed an upregulation somewhat specific to cytosolic HSPs, whereas little change was detected among HSPs predominately located in the mitochondria and ER. This was a surprising observation considering TFEC damage likely originates from within the mitochondrial matrix.

3.3. Flutamide. Flutamide is an antiandrogen agent used for the treatment of metastatic prostate cancer; however, flutamide is also an idiosyncratic hepatotoxicant, inducing liver dysfunction in 1-10\% of users [57-59]. A study investigating the effect of flutamide on rat hepatocytes proposed the toxicity is due to metabolite formation by cytochrome P450 and inhibition of mitochondrial respiration [60]. A structuretoxicity relationship with a nitro cyano analog of flutamide (CYA) has been used to differentiate hepatocyte viability and pinpoint mechanistic differences in cells exposed to these agents, since nitroaromatic groups have been associated with idiosyncratic toxicity [61]. In TAMH, flutamide was shown to be approximately two times as toxic compared to CYA, which was accompanied by greater upregulation of $\mathrm{Nrf} 2$ responsive genes following flutamide exposure (Figure 1(c)) [61]. Additionally, flutamide altered mitochondrial morphology and profoundly downregulated genes associated with fatty acid $\beta$-oxidation and upregulated genes related to antioxidant defense. Loss of ATP was also found to be a critical element in flutamide cytotoxicity caused by its ability to target complex I of the electron transport chain and impair oxidative phosphorylation. When the potency of complex I inhibition was analyzed using rotenone, a known complex I inhibitor, a higher level of inhibition was observed. Rotenone exposure results in vacuous mitochondria with cristae barely intact, whereas flutamide exposed cells had either electron dense 
or visibly swollen, vacuous mitochondria. The observation that rotenone is a more potent inhibitor, but seemingly less cytotoxic, suggests other factors beyond complex I inhibition likely play a role in flutamide-induced toxicity.

3.4. PD0325901. PD0325901 is an agent capable of blocking conversion of ERK to its activated, phosphorylated state (p-ERK) by inhibiting activated mitogen-activated protein kinase 1/2 (MEK1/2), an upstream kinase in the ERK1/2 phosphorelay cascade [62]. The ERK pathway is upregulated in most human hepatocellular carcinoma and is therefore a target for many chemotherapy regimens [20]; therefore, there has been interest in evaluating PD0325901 both in vivo and in vitro $[63,64]$. In TAMH cells specifically, p-ERK protein levels were shown to decrease with increasing concentrations of PD325901 (Figure 1(d)) [63]. In fact, PD0325901 was shown to be effective in reducing p-ERK levels and cell growth in TAMH at nanomolar concentrations. In the same study, reduction of p-ERK, as well as tumor growth rate, was observed in PD0325901-treated athymic mice with TAMH flank tumors. To evaluate inhibition of the ERK pathway in vivo, the transgenic mice from which the TAMH line was developed demonstrated decreased incidence of hepatocellular carcinoma after exposure to the drug. Overall, PD0325901 induced apoptosis and reduction in tumor growth, which resulted in regression but not lack of tumor presence. A pilot study was conducted utilizing this agent as a possible chemotherapy for 13 patients with advanced breast cancer, melanoma, and colon cancer [64]. Transitioning PD0325901 treatment from the TAMH model to humans showed some similarities in that PD0325901 was able to reduce tumor growth. However, complications related to the complexities of human metabolism and adverse events led to the eventual termination of the study.

3.5. Lapatinib. Lapatinib is an orally administered tyrosine kinase inhibitor and is proven to inhibit the proliferation of tumor cells that overexpress epidermal growth factor receptors (EGFRs) [65]. Previously existing clinical evidence indicates tyrosine kinase inhibitors such as lapatinib might be associated with idiosyncratic hepatotoxicity through generation of a reactive metabolite [66]. A case control study evaluating data of 120 patients indicated that the effect of altering metabolism and hepatotoxicity by addition of dexamethasone, a CYP3A4 inducer, differs between individuals due to genetic differences [67]. In TAMH, concomitant exposure to lapatinib and dexamethasone increased the risk of hepatotoxicity fivefold. Lapatinib is a CYP3A4 substrate; therefore, when used concomitantly with dexamethasone there is an increase in the potential risk for toxicity due to increased reactive metabolite formation. Exposure to both agents in TAMH cells caused a large reduction in viability as compared to treatment with lapatinib alone due to dexamethasone's ability to induce CYP3A4. These results caution that inducers or inhibitors of this CYP450 isoform may increase the risk of hepatotoxicity when coadministered with lapatinib and should be used cautiously when treating cancer patients.
3.6. Flupirtine. Flupirtine is a central acting analgesic offered in European countries and Brazil as an alternative to nonopioid anti-inflammatory drugs [68]. The mechanism of flupirtine action is thought to be based on the drug's ability to open voltage gated potassium channels in the central nervous system [69]. Administration of flupirtine has been associated with hepatocellular injury and is thought to be related to flupirtine metabolism, which has previously been shown to undergo both oxidative and conjugative reactions in vivo and in vitro [70-72]. Oxidation of flupirtine is performed by peroxidases to form quinone diimine metabolites; however, oxidation potentials and toxicity were not correlated in studies using the TAMH line [72]. When assessing hepatotoxicity of flupirtine in vivo, a double-blind study was conducted in which a group of patients received clinically relevant doses for either four or eight weeks [70]. Of the 207 patients participating in the trial, eight individuals showed critically higher levels of liver enzymes. Furthermore, seven of these eight patients were receiving flupirtine. This link led to immediate termination of the clinical trial. Elevated liver enzymes were also seen in a small percentage of patients in a separate clinical study [73]. Flupirtine's reactive quinone diimine metabolite resembles the reactive quinoneimine metabolite (NAPQI) that is generated during APAP oxidation [71]. However, differences in physiochemical properties between APAP (a hydrophilic, weak acid) and flupirtine (a lipophilic, weak base) likely contribute to the observed differences in hepatocyte uptake and compartmentalization of these agents [72]. In the case of flupirtine, unlike acetaminophen, hepatotoxicity was not directly linked to oxidative processes. Based on the studies in TAMH it was postulated that redox behavior does not play a central role in flupirtine hepatotoxicity; rather oxidation potential seemed to correlate closely with channelopening activity.

\section{Conclusions}

The TAMH cell line, which overexpresses human TGF- $\alpha$, is a model that utilizes many biochemical processes altered by heptatotoxicants during drug-induced liver injury in vivo. This cell line retains a stable phenotype and expresses drugmetabolizing enzymes without the limits of using cultured human primary hepatocytes or human hepatoma cell lines (e.g., HepG2) [74, 75]. Still, many of the same pathways affected by APAP, TFEC, flutamide, PD0325901, lapatinib, and flupirtine in human hepatocytes are similarly altered in the TAMH model. The toxicity associated with many of these agents is thought to be mediated in part through reactive metabolite formation. The TAMH line retains $\mathrm{P} 450$ protein expression capable of forming these metabolites as evidenced by studies with APAP, TFEC, and lapatinib. Reactive metabolites from both APAP and TFEC have been shown to generate protein adducts in TAMH. The formation of these adducts following flutamide, lapatinib, or flupirtine exposure has yet to be investigated in TAMH, but GSHadducts have been reported in liver microsomes exposed to these agents $[66,71,76]$. Toxicity associated with APAP, TFEC, and flutamide led to decreased ATP production, with TFEC and flutamide also initiating upregulation of Nrf2 and 
antioxidant defense genes. To our knowledge, APAP-induced changes in Nrf2 levels and subcellular localization have yet to be investigated in TAMH. Interestingly, TAMH may also be able to differentiate between hepatocellular injury associated with oxidative stress and toxicity resulting from nonoxidative processes as in the case of flupirtine [72].

TAMH has been shown to possess mechanistic qualities that serve functional roles during hepatocellular injury in vivo such as GSH, Nrf2, Bcl-2, and MAPK signaling. Confirmation of these pathways only represents a small percentage of the mechanistic complexity associated with drug-induced hepatocellular injury, especially in relation to oxidative stress. Future work should be done to characterize the roles of enzymes involved in ROS detoxification such as catalase, superoxide dismutase, and glutathione peroxidase. Based on the fact that reactive metabolite formation and/or oxidative stress have been implicated in toxicity associated with APAP, TFEC, lapatinib, flutamide, and flupirtine, further investigations into how these protective enzymes are affected in the TAMH line are warranted. This is especially true considering the activity of these enzymes has already been investigated and characterized using in vivo systems during APAPinduced liver injury [77]. Based on the immortalized nature of the TAMH line, studies investigating cell proliferation or carcinogenesis might find utility in this model. PD0325901 was shown to reduce p-ERK levels leading to decreased cell growth in TAMH similar to in vivo systems [63].

Future initiatives in TAMH should further characterize the role protective enzymes play in the detoxification of hepatotoxicant-induced ROS production. In fact, altered expression of oxidative stress-related proteins and proteins associated with mitochondrial respiration and metabolism have been shown to be consistent biomarkers for hepatotoxicity in rats across in vitro and in vivo systems [78]. Moreover, comparing other in vitro models of hepatotoxicity with TAMH should provide insight for researchers in selecting the specific in vitro system for their research. While murine models share many similarities with human ones, it is worth noting that disparities between the two species are evident. For example, differences in the timing of cell death and possible cell signaling mechanisms have been reported during exposure to toxicologically relevant APAP concentrations [79]. TAMH by no means represents a perfect tool for correlating in vitro drug response to the in vivo condition. In vitro models possess inherent drawbacks as predictive models [80], challenges that are evident in TAMH from specific studies previously discussed herein involving PD0325901 and APAP. Complexities associated with drug absorption, distribution, metabolism, excretion, and transport in vivo are challenging to replicate in vitro. That being said, the ability of the TAMH cell line to maintain drug-metabolizing enzyme expression and retain a stable phenotype with intact and suitable mechanistic capabilities makes it a useful in vitro model for assessing drug-induced hepatocellular injury.

\section{Competing Interests}

The authors declare that there is no conflict of interests regarding the publication of this paper.

\section{References}

[1] J. C. Wu, G. Merlino, K. Cveklova, B. Mosinger Jr., and N. Fausto, "Autonomous growth in serum-free medium and production of hepatocellular carcinomas by differentiated hepatocyte lines that overexpress transforming growth factor alpha 1," Cancer Research, vol. 54, no. 22, pp. 5964-5973, 1994.

[2] N. Fausto, "Growth factors in liver development, regeneration and carcinogenesis," Progress in Growth Factor Research, vol. 3, no. 3, pp. 219-234, 1991.

[3] J. T. Brooling, J. S. Campbell, C. Mitchell, G. C. Yeoh, and N. Fausto, "Differential regulation of rodent hepatocyte and oval cell proliferation by interferon $\gamma$," Hepatology, vol. 41, no. 4, pp. 906-915, 2005.

[4] L. D. DeLeve and N. Kaplowitz, "Glutathione metabolism and its role in hepatotoxicity," Pharmacology and Therapeutics, vol. 52, no. 3, pp. 287-305, 1991.

[5] O. W. Griffith and R. T. Mulcahy, "The enzymes of glutathione synthesis: $\gamma$-glutamylcysteine synthetase," Advances in Enzymology and Related Areas of Molecular Biology, vol. 73, pp. 209267, 1999.

[6] C. C. Franklin, M. E. Rosenfeld-Franklin, C. White, T. J. Kavanagh, and N. Fausto, "TGFbetal-induced suppression of glutathione antioxidant defenses in hepatocytes: caspasedependent post-translational and caspase-independent transcriptional regulatory mechanisms," The FASEB Journal, vol. 17, no. 11, pp. 1535-1537, 2003.

[7] L. Braun, J. E. Mead, M. Panzica, R. Mikumo, G. I. Bell, and N. Fausto, "Transforming growth factor $\beta$ mRNA increases during liver regeneration: a possible paracrine mechanism of growth regulation," Proceedings of the National Academy of Sciences of the United States of America, vol. 85, no. 5, pp. 1539-1543, 1988.

[8] R. H. Pierce, J. S. Campbell, A. B. Stephenson et al., "Disruption of redox homeostasis in tumor necrosis factor-induced apoptosis in a murine hepatocyte cell line," American Journal of Pathology, vol. 157, no. 1, pp. 221-236, 2000.

[9] C. M. Knudson and S. J. Korsmeyer, "Bcl-2 and bax function independently to regulate cell death," Nature Genetics, vol. 16, no. 4, pp. 358-363, 1997.

[10] H. Jaeschke and M. L. Bajt, "Intracellular signaling mechanisms of acetaminophen-induced liver cell death," Toxicological Sciences, vol. 89, no. 1, pp. 31-41, 2006.

[11] A. Hamid Boulares, A. J. Zoltoski, B. A. Stoica, O. Cuvillier, and M. E. Smulson, "Acetaminophen induces a caspase-dependent and Bcl-xL sensitive apoptosis in human hepatoma cells and lymphocytes," Pharmacology and Toxicology, vol. 90, no. 1, pp. 38-50, 2002.

[12] H. K. Ho, Z.-H. Hu, S.-P. Tzung et al., "BCL-xL overexpression effectively protects against tetrafluoroethylcysteineinduced intramitochondrial damage and cell death," Biochemical Pharmacology, vol. 69, no. 1, pp. 147-157, 2005.

[13] P. S. Schwartz, M. K. Manion, C. B. Emerson et al., "2-Methoxy antimycin reveals a unique mechanism for Bcl- $\mathrm{x}_{L}$ inhibition," Molecular Cancer Therapeutics, vol. 6, no. 7, pp. 2073-2080, 2007.

[14] H. Jaeschke, M. R. McGill, and A. Ramachandran, "Oxidant stress, mitochondria, and cell death mechanisms in druginduced liver injury: lessons learned from acetaminophen hepatotoxicity," Drug Metabolism Reviews, vol. 44, no. 1, pp. 88106, 2012

[15] T. W. Kensler, N. Wakabayashi, and S. Biswal, "Cell survival responses to environmental stresses via the Keap1-Nrf2-ARE 
pathway," Annual Review of Pharmacology and Toxicology, vol. 47, pp. 89-116, 2007.

[16] Z. Sun, Z. Huang, and D. D. Zhang, "Phosphorylation of Nrf2 at multiple sites by MAP kinases has a limited contribution in modulating the Nrf2-dependent antioxidant response," PLoS ONE, vol. 4, no. 8, Article ID e6588, 2009.

[17] K. Itoh, K. I. Tong, and M. Yamamoto, "Molecular mechanism activating Nrf2-Keap1 pathway in regulation of adaptive response to electrophiles," Free Radical Biology and Medicine, vol. 36, no. 10, pp. 1208-1213, 2004.

[18] G. L. Johnson, H. G. Dohlman, and L. M. Graves, "MAPK kinase kinases (MKKKs) as a target class for small-molecule inhibition to modulate signaling networks and gene expression," Current Opinion in Chemical Biology, vol. 9, no. 3, pp. 325-331, 2005.

[19] A. G. Turjanski, J. P. Vaqué, and J. S. Gutkind, "MAP kinases and the control of nuclear events," Oncogene, vol. 26, no. 22, pp. 3240-3253, 2007.

[20] I. H. McKillop, C. M. Schmidt, P. A. Cahill, and J. V. Sitzmann, "Altered expression of mitogen-activated protein kinases in a rat model of experimental hepatocellular carcinoma," Hepatology, vol. 26, no. 6, pp. 1484-1491, 1997.

[21] S. Osada, M. Kanematsu, H. Imai, S. Goshima, and Y. Sugiyama, "Evaluation of extracellular signal regulated kinase expression and its relation to treatment of hepatocellular carcinoma," Journal of the American College of Surgeons, vol. 201, no. 3, pp. 405-411, 2005.

[22] C. M. Schmidt, I. H. McKillop, P. A. Cahill, and J. V. Sitzmann, "Increased MAPK expression and activity in primary human hepatocellular carcinoma," Biochemical and Biophysical Research Communications, vol. 236, no. 1, pp. 54-58, 1997.

[23] Y. Tsuboi, T. Ichida, S. Sugitani et al., "Overexpression of extracellular signal-regulated protein kinase and its correlation with proliferation in human hepatocellular carcinoma," Liver International, vol. 24, no. 5, pp. 432-436, 2004.

[24] G. H. Thoresen, T. K. Guren, D. Sandnes, M. Peak, L. Agius, and T. Christoffersen, "Response to transforming growth factor alpha (TGFalpha) and epidermal growth factor (EGF) in hepatocytes: lower EGF receptor affinity of TGFalpha is associated with more sustained activation of p42/p44 mitogen-activated protein kinase and greater efficacy in stimulation of DNA synthesis," Journal of Cellular Physiology, vol. 175, no. 1, pp. 10$18,1998$.

[25] B. L. Bennett, D. T. Sasaki, B. W. Murray et al., "SP600125, an anthrapyrazolone inhibitor of Jun N-terminal kinase," Proceedings of the National Academy of Sciences of the United States of America, vol. 98, no. 24, pp. 13681-13686, 2001.

[26] C. Latchoumycandane, C. W. Goh, M. M. K. Ong, and U. A. Boelsterli, "Mitochondrial protection by the JNK inhibitor leflunomide rescues mice from acetaminophen-induced liver injury," Hepatology, vol. 45, no. 2, pp. 412-421, 2007.

[27] W. M. Lee, "Acetaminophen and the U.S. acute liver failure study group: lowering the risks of hepatic failure," Hepatology, vol. 40, no. 1, pp. 6-9, 2004.

[28] P. Nourjah, S. R. Ahmad, C. Karwoski, and M. Willy, "Estimates of acetaminophen (paracetomal)-associated overdoses in the United States," Pharmacoepidemiology and Drug Safety, vol. 15, no. 6, pp. 398-405, 2006.

[29] J. A. Hinson, D. W. Roberts, and L. P. James, "Mechanisms of acetaminophen-induced liver necrosis," in Handbook of Experimental Pharmacology, vol. 196, pp. 369-405, 2010.

[30] S. D. Nelson, M. A. Tirmenstein, M. S. Rashed, and T. G. Myers, "Acetaminophen and protein thiol modification," in Biological
Reactive Intermediates IV, vol. 283 of Advances in Experimental Medicine and Biology, pp. 579-588, Springer, New York, NY, USA, 1991.

[31] R. H. Pierce, C. C. Franklin, J. S. Campbell et al., "Cell culture model for acetaminophen-induced hepatocyte death in vivo," Biochemical Pharmacology, vol. 64, no. 3, pp. 413-424, 2002.

[32] W. Chen, L. L. Koenigs, S. J. Thompson et al., "Oxidation of acetaminophen to its toxic quinone imine and nontoxic catechol metabolites by baculovirus-expressed and purified human cytochromes P450 2E1 and 2A6," Chemical Research in Toxicology, vol. 11, no. 4, pp. 295-301, 1998.

[33] E. B. Nelson, "The pharmacology and toxicology of metasubstituted acetanilide I: acute toxicity of 3-hydroxyacetanilide in mice," Research Communications in Chemical Pathology and Pharmacology, vol. 28, no. 3, pp. 447-456, 1980.

[34] T. G. Myers, E. C. Dietz, N. L. Anderson, E. A. Khairallah, S. D. Cohen, and S. D. Nelson, "A comparative study of mouse liver proteins arylated by reactive metabolites of acetaminophen and its nonhepatotoxic regioisomer, 3 '-hydroxyacetanilide," Chemical Research in Toxicology, vol. 8, no. 3, pp. 403-413, 1995.

[35] Y. Qiu, L. Z. Benet, and A. L. Burlingame, "Identification of hepatic protein targets of the reactive metabolites of the nonhepatotoxic regioisomer of acetaminophen, $3^{\prime}$-hydroxyacetanilide, in the mouse in vivo using two-dimensional gel electrophoresis and mass spectrometry," Advances in Experimental Medicine and Biology, vol. 500, pp. 663-673, 2001.

[36] W. F. Salminen Jr., S. M. Roberts, N. R. Pumford, and J. A. Hinson, "Immunochemical comparison of 3 '-hydroxyacetanilide and acetaminophen binding in mouse liver," Drug Metabolism and Disposition, vol. 26, no. 3, pp. 267-271, 1998.

[37] M. A. Tirmenstein and S. D. Nelson, "Subcellular binding and effects on calcium homeostasis produced by acetaminophen and a nonhepatotoxic regioisomer, 3'-hydroxyacetanilide, in mouse liver," Journal of Biological Chemistry, vol. 264, no. 17, pp. 9814-9819, 1989.

[38] Y. Xie, M. R. McGill, K. Du et al., "Mitochondrial protein adducts formation and mitochondrial dysfunction during $\mathrm{N}$ acetyl-m-aminophenol (AMAP)-induced hepatotoxicity in primary human hepatocytes," Toxicology and Applied Pharmacology, vol. 289, no. 2, pp. 213-222, 2015.

[39] B. D. Stamper, I. Mohar, T. J. Kavanagh, and S. D. Nelson, "Proteomic analysis of acetaminophen-induced changes in mitochondrial protein expression using spectral counting," Chemical Research in Toxicology, vol. 24, no. 4, pp. 549-558, 2011.

[40] B. D. Stamper, T. K. Bammler, R. P. Beyer, F. M. Farin, and S. D. Nelson, "Differential regulation of mitogen-activated protein kinase pathways by acetaminophen and its nonhepatotoxic regioisomer $3^{\prime}$-hydroxyacetanilide in TAMH cells," Toxicological Sciences, vol. 116, no. 1, pp. 164-173, 2010.

[41] K. Kon, J.-S. Kim, A. Uchiyama, H. Jaeschke, and J. J. Lemasters, "Lysosomal iron mobilization and induction of the mitochondrial permeability transition in acetaminophen-induced toxicity to mouse hepatocytes," Toxicological Sciences, vol. 117, no. 1, pp. 101-108, 2010.

[42] R. R. Ramsay, M. S. Rashed, and S. D. Nelson, "In vitro effects of acetaminophen metabolites and analogs on the respiration of mouse liver mitochondria," Archives of Biochemistry and Biophysics, vol. 273, no. 2, pp. 449-457, 1989.

[43] T. Hai, C. D. Wolfgang, D. K. Marsee, A. E. Allen, and U. Sivaprasad, "ATF3 and stress responses," Gene Expression, vol. 7, no. 4-6, pp. 321-335, 1999. 
[44] B. D. Stamper, M. L. Garcia, D. Q. Nguyen et al., "P53 contributes to differentiating gene expression following exposure to acetaminophen and its less hepatotoxic regioisomer both in vitro and in vivo," Gene Regulation and Systems Biology, vol. 9, pp. 1-14, 2015.

[45] A. V. Vaseva and U. M. Moll, "The mitochondrial p53 pathway," Biochimica et Biophysica Acta (BBA)_Bioenergetics, vol. 1787, no. 5, pp. 414-420, 2009.

[46] Y. Jiang, X. Fan, Y. Wang et al., "Schisandrol B protects against acetaminophen-induced hepatotoxicity by inhibition of CYPmediated bioactivation and regulation of liver regeneration," Toxicological Sciences, vol. 143, no. 1, pp. 107-115, 2015.

[47] Y.-S. Lee, J. Wan, B.-J. Kim, M.-A. Bae, and B. J. Song, "Ubiquitin-dependent degradation of $\mathrm{p} 53$ protein despite phosphorylation at its $\mathrm{N}$ terminus by acetaminophen," Journal of Pharmacology and Experimental Therapeutics, vol. 317, no. 1, pp. 202-208, 2006.

[48] K. Du, Y. Xie, M. R. McGill, and H. Jaeschke, "Pathophysiological significance of c-jun N-terminal kinase in acetaminophen hepatotoxicity," Expert Opinion on Drug Metabolism and Toxicology, vol. 11, no. 11, pp. 1769-1779, 2015.

[49] C. Saito, J. J. Lemasters, and H. Jaeschke, "C-Jun N-terminal kinase modulates oxidant stress and peroxynitrite formation independent of inducible nitric oxide synthase in acetaminophen hepatotoxicity," Toxicology and Applied Pharmacology, vol. 246, no. 1-2, pp. 8-17, 2010.

[50] N. Hanawa, M. Shinohara, B. Saberi, W. A. Gaarde, D. Han, and N. Kaplowitz, "Role of JNK translocation to mitochondria leading to inhibition of mitochondria bioenergetics in acetaminophen-induced liver injury," The Journal of Biological Chemistry, vol. 283, no. 20, pp. 13565-13577, 2008.

[51] E. A. Lock and J. Ishmael, "The nephrotoxicity and hepatotoxicity of 1,1,2,2-tetrafluoroethyl-L-cysteine in the rat," Archives of Toxicology, vol. 72, no. 6, pp. 347-354, 1998.

[52] J. Odum and T. Green, "The metabolism and nephrotoxicity of tetrafluoroethylene in the rat," Toxicology and Applied Pharmacology, vol. 76, no. 2, pp. 306-318, 1984.

[53] A. J. L. Cooper and J. T. Pinto, "Aminotransferase, L-amino acid oxidase and $\beta$-lyase reactions involving $\mathrm{L}$-cysteine $\mathrm{S}$-conjugates found in allium extracts: relevance to biological activity?" Biochemical Pharmacology, vol. 69, no. 2, pp. 209-220, 2005.

[54] E. A. James, S. P. Gygi, M. L. Adams et al., "Mitochondrial aconitase modification, functional inhibition, and evidence for a supramolecular complex of the TCA cycle by the renal toxicant S-(1,1,2,2-tetrafluoroethyl)-L-cysteine," Biochemistry, vol. 41, no. 21, pp. 6789-6797, 2002.

[55] H. K. Ho, Y. Jia, K. J. Coe et al., "Cytosolic heat shock proteins and heme oxygenase-1 are preferentially induced in response to specific and localized intramitochondrial damage by tetrafluoroethylcysteine," Biochemical Pharmacology, vol. 72, no. 1, pp. 80-90, 2006.

[56] T. Bammler, R. P. Beyer, and S. Bhattacharya, "Standardizing global gene expression analysis between laboratories and across platforms," Nature Methods, vol. 2, no. 5, pp. 351-356, 2005.

[57] M. García Cortés, R. J. Andrade, M. I. Lucena et al., "Flutamideinduced hepatotoxicity: report of a case series," Revista Espanola de Enfermedades Digestivas, vol. 93, no. 7, pp. 423-432, 2001.

[58] J.-L. Gomez, A. Dupont, L. Cusan et al., "Incidence of liver toxicity associated with the use of flutamide in prostate cancer patients," The American Journal of Medicine, vol. 92, no. 5, pp. 465-470, 1992.
[59] D. K. Wysowski and J. L. Fourcroy, "Flutamide hepatotoxicity," The Journal of Urology, vol. 155, no. 1, pp. 209-212, 1996.

[60] D. Fau, D. Eugene, A. Berson et al., "Toxicity of the antiandrogen flutamide in isolated rat hepatocytes," Journal of Pharmacology and Experimental Therapeutics, vol. 269, no. 3, pp. 954-962, 1994.

[61] K. J. Coe, Y. Jia, K. H. Han et al., "Comparison of the cytotoxicity of the nitroaromatic drug flutamide to its cyano analogue in the hepatocyte cell line TAMH: evidence for complex I inhibition and mitochondrial dysfunction using toxicogenomic screening," Chemical Research in Toxicology, vol. 20, no. 9, pp. 1277-1290, 2007.

[62] S. D. Barrett, A. J. Bridges, D. T. Dudley et al., “The discovery of the benzhydroxamate MEK inhibitors CI-1040 and PD 0325901," Bioorganic and Medicinal Chemistry Letters, vol. 18, no. 24, pp. 6501-6504, 2008.

[63] M. Hennig, M. T. Yip-Schneider, S. Wentz et al., “Targeting mitogen-activated protein Kinase Kinase with the inhibitor pd0325901 decreases hepatocellular carcinoma growth in vitro and in mouse model systems," Hepatology, vol. 51, no. 4, pp. 1218-1225, 2010.

[64] P. D. Boasberg, C. H. Redfern, G. A. Daniels, D. Bodkin, C. R. Garrett, and A. D. Ricart, "Pilot study of PD-0325901 in previously treated patients with advanced melanoma, breast cancer, and colon cancer," Cancer Chemotherapy and Pharmacology, vol. 68, no. 2, pp. 547-552, 2011.

[65] W. Xia, R. J. Mullin, B. R. Keith et al., "Anti-tumor activity of GW572016: a dual tyrosine kinase inhibitor blocks EGF activation of EGFR/erbB2 and downstream Erk1/2 and AKT pathways," Oncogene, vol. 21, no. 41, pp. 6255-6263, 2002.

[66] W. C. Teng, J. W. Oh, L. S. New et al., "Mechanism-based inactivation of cytochrome P450 3A4 by lapatinib," Molecular Pharmacology, vol. 78, no. 4, pp. 693-703, 2010.

[67] Y. L. Teo, M. Saetaew, S. Chanthawong et al., "Effect of CYP3A4 inducer dexamethasone on hepatotoxicity of lapatinib: clinical and in vitro evidence," Breast Cancer Research and Treatment, vol. 133, no. 2, pp. 703-711, 2012.

[68] H. A. Friedel and A. Fitton, "Flupirtine. A review of its pharmacological properties, and therapeutic efficacy in pain states," Drugs, vol. 45, no. 4, pp. 548-569, 1993.

[69] I. Szelenyi, "Flupirtine, a re-discovered drug, revisited," Inflammation Research, vol. 62, no. 3, pp. 251-258, 2013.

[70] M. C. Michel, P. Radziszewski, C. Falconer, D. MarschallKehrel, and K. Blot, "Unexpected frequent hepatotoxicity of a prescription drug, flupirtine, marketed for about 30 years," British Journal of Clinical Pharmacology, vol. 73, no. 5, pp. 821825, 2012.

[71] K. Methling, P. Reszka, M. Lalk et al., "Investigation of the in vitro metabolism of the analgesic flupirtine," Drug Metabolism and Disposition, vol. 37, no. 3, pp. 479-493, 2009.

[72] C. J. Lemmerhirt, M. Rombach, A. Bodtke, P. J. Bednarski, and A. Link, "Oxidation potentials of N-modified derivatives of the analgesic flupirtine linked to potassium $\mathrm{K}_{\mathrm{V}} 7$ channel opening activity but not hepatocyte toxicity," ChemMedChem, vol. 10, no. 2, pp. 368-379, 2015.

[73] C. Li, J. Ni, Z. Wang et al., "Analgesic efficacy and tolerability of flupirtine vs. tramadol in patients with subacute low back pain: a double-blind multicentre trial," Current Medical Research and Opinion, vol. 24, no. 12, pp. 3523-3530, 2008.

[74] M. J. Gomez-Lechon, M. T. Donato, J. V. Castell, and R. Jover, "Human hepatocytes as a tool for studying toxicity and drug 
metabolism," Current Drug Metabolism, vol. 4, no. 4, pp. 292312, 2003.

[75] M. Vermeir, P. Annaert, R. N. V. S. Mamidi, D. Roymans, W. Meuldermans, and G. Mannens, "Cell-based models to study hepatic drug metabolism and enzyme induction in humans," Expert Opinion on Drug Metabolism and Toxicology, vol. 1, no. 1, pp. 75-90, 2005.

[76] P. Kang, D. Dalvie, E. Smith, S. Zhou, and A. Deese, "Identification of a novel glutathione conjugate of flutamide in incubations with human liver microsomes," Drug Metabolism and Disposition, vol. 35, no. 7, pp. 1081-1088, 2007.

[77] S. L. Arnaiz, S. Llesuy, J. C. Cutrín, and A. Boveris, "Oxidative stress by acute acetaminophen administration in mouse-liver," Free Radical Biology and Medicine, vol. 19, no. 3, pp. 303-310, 1995.

[78] R. Kikkawa, M. Fujikawa, T. Yamamoto, Y. Hamada, H. Yamada, and I. Horii, "In vivo hepatotoxicity study of rats in comparison with in vitro hepatotoxicity screening system," Journal of Toxicological Sciences, vol. 31, no. 1, pp. 23-34, 2006.

[79] Y. Xie, M. R. McGill, K. Dorko et al., "Mechanisms of acetaminophen-induced cell death in primary human hepatocytes," Toxicology and Applied Pharmacology, vol. 279, no. 3, pp. 266-274, 2014.

[80] A. Costa, B. Sarmento, and V. Seabra, "An evaluation of the latest in vitro tools for drug metabolism studies," Expert Opinion on Drug Metabolism and Toxicology, vol. 10, no. 1, pp. 103-119, 2014.

[81] H. K. Ho, C. C. White, C. Fernandez et al., "Nrf2 activation involves an oxidative-stress independent pathway in tetrafluoroethylcysteine-induced cytotoxicity," Toxicological Sciences, vol. 86, no. 2, pp. 354-364, 2005. 

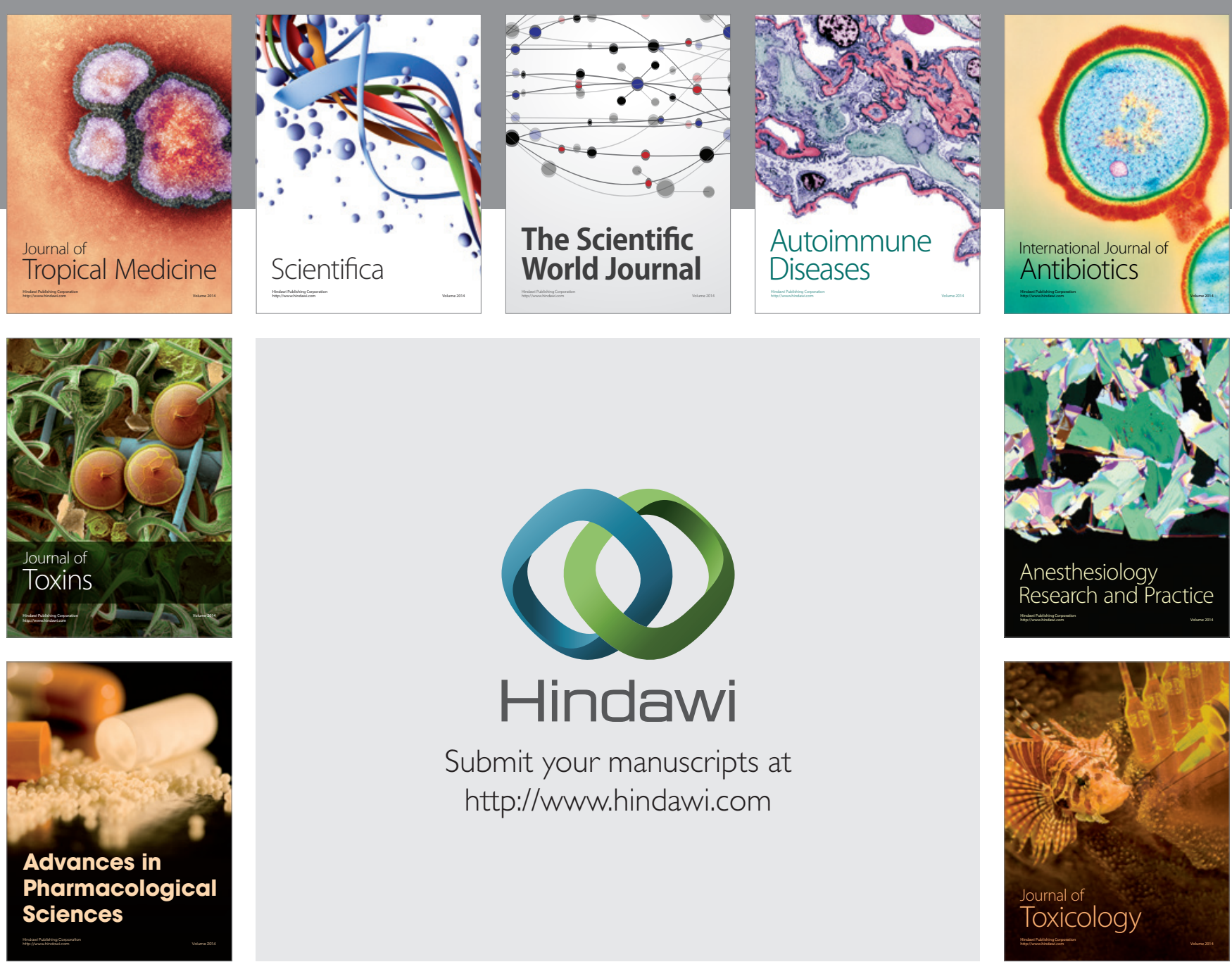

\section{Hindawi}

Submit your manuscripts at

http://www.hindawi.com
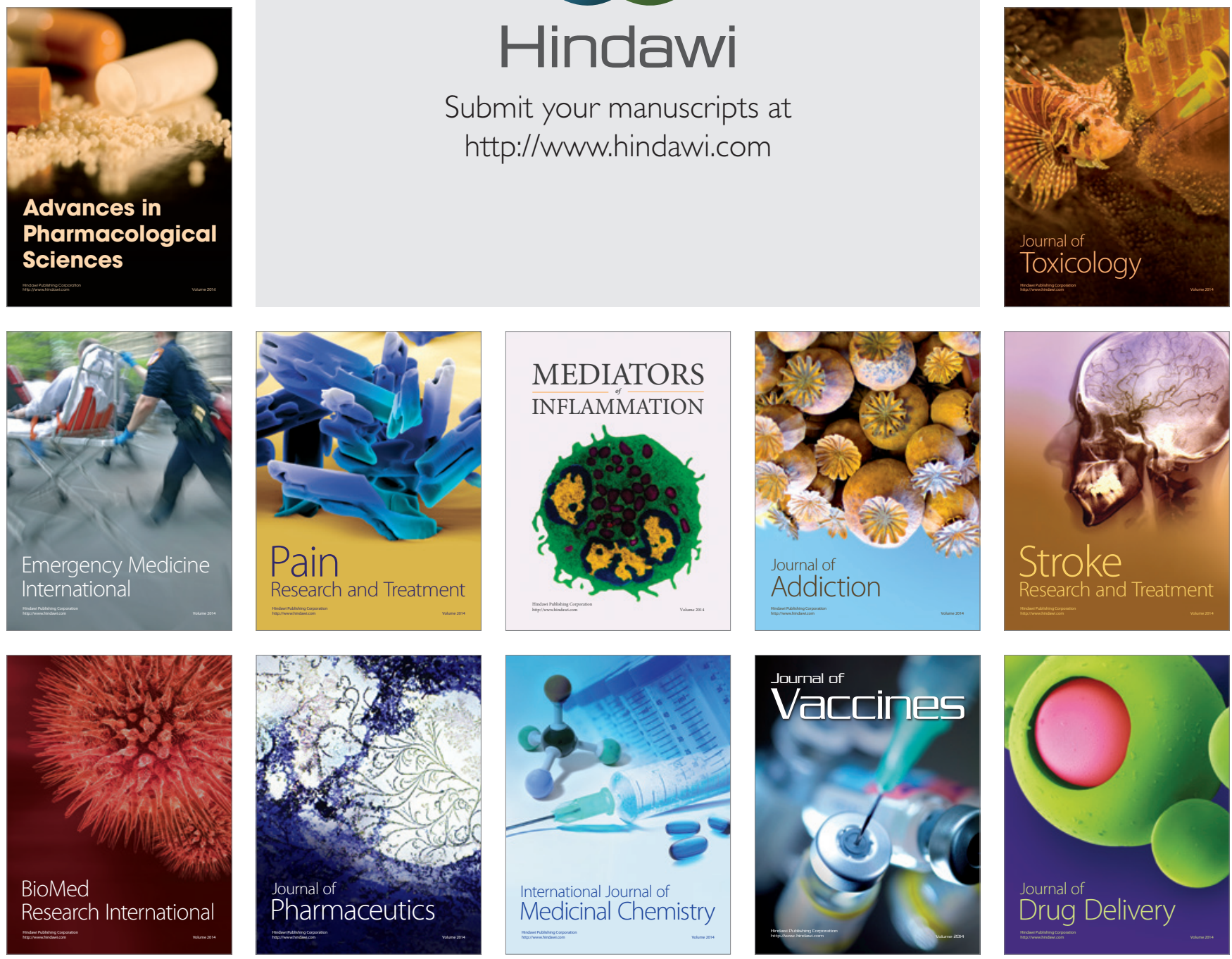